\title{
Characteristics of Laser Backscattering Intensity to Detect Frozen and Wet Surfaces on Roads
}

\author{
Jungil Shin $\mathbb{D}^{1},{ }^{1}$ Hyunsuk Park, ${ }^{2}$ and Taejung Kim ${ }^{3}$ \\ ${ }^{1}$ Research Center of Geoinformatic Engineering, Inha University, Incheon 22212, Republic of Korea \\ ${ }^{2}$ Department of Future Technology and Convergence Research, Korea Institute of Civil Engineering and Building Technology, \\ Goyang 10223, Republic of Korea \\ ${ }^{3}$ Department of Geoinformatic Engineering, Inha University, Incheon 22212, Republic of Korea \\ Correspondence should be addressed to Jungil Shin; jishin@inha.ac.kr
}

Received 1 February 2019; Revised 28 March 2019; Accepted 1 April 2019; Published 17 April 2019

Guest Editor: Sang-Hoon Hong

Copyright (C) 2019 Jungil Shin et al. This is an open access article distributed under the Creative Commons Attribution License, which permits unrestricted use, distribution, and reproduction in any medium, provided the original work is properly cited.

\begin{abstract}
A frozen or wet road surface is a cause of skidding and accidents, so road surface condition is important information for driving safety. Some instruments and methods have been developed to investigate road surface conditions based on optical imagery, although an active sensor is needed, regardless of the time of day. Recently, the laser scanner, which acquires backscattering intensity data related to reflectivity, has become popular in various fields. There is a need to investigate road surface conditions (frozen, wet, or dry) using laser backscattering intensity. This study tries to analyze signal characteristics of laser backscattering intensity to detect frozen and wet surfaces on roads. An ice target with a $7 \mathrm{~cm}$ thickness was placed on a road surface, and a wet surface was made due to the melting ice. The ice target, wet surface, dry surface, and roadside vegetation were scanned using a laser scanner. As a result, backscattering signals from the top surface of the ice target were missing due to its smoothness. Dry and wet asphalt surfaces showed distinguishable intensity ranges in their signals. The thick sidewall of the ice target and vegetation at the roadside showed overlapping intensity ranges. An ice sheet is only a few millimeters thick on a real road surface, and the roadside vegetation might be easily distinguished by using texture or auxiliary data. Therefore, laser backscattering intensity can be used to detect frozen, wet, and dry road surfaces, regardless of the time of day. The laser scanner can be installed to acquire information about road surface conditions from observation stations and vehicles in an application for transportation.
\end{abstract}

\section{Introduction}

Road surface condition is important information for driving safety. The road surface condition due to weather has been reported as the primary reason for traffic accidents [1-3]. Frozen and wet conditions cause skidding and subsequent accidents due to the unexpected loss of traction. In particular, the rate of traffic accidents is very high at night under frozen conditions [4]. In the dark, a frozen area of a wet road surface (called black ice) is generally invisible to drivers. Black ice is a thin coating of glaze ice on a surface, especially on roads [5]. The ice itself is not black but is visually transparent, so only the black road below can be seen through it.

Several methods have been developed to investigate various conditions on road surfaces. Equipment was developed to measure the condition of the road surface using a near-infrared (NIR) light source and a detector mounted on the front of a vehicle [6]. Many studies that tried to classify road surface conditions (such as dry, wet, and icy) using visible or NIR imagery showed high classification accuracies [7-14]. Recently, a system was commercialized for analysis of the conditions on road and runway surfaces. It consists of a camera using light-emitting diode (LED) lighting and various weather sensors [15]. In addition, active sensor technologies have been developed to classify road surface conditions using backscattering signals from radar and sonar [16]. Most of these studies focused on analyzing various road conditions using imagery from optical sensors. However, the passive optical sensor has limited use in the dark, even though it can use artificial light. Therefore, it is necessary to develop a detection method for black ice and wet road conditions, regardless of the time of day, because the probability of 
an accident from frozen and wet surfaces is higher at night.

Radar and light detection and ranging (LiDAR) are representative active sensors. They can be used regardless of the time of day, because they have both an energy source and a detector in a sensor system. Active sensors have limited use due to their high price, despite the enhancement of manufacturing and application technology [17]. Recently, the laser scanner has become popular owing to miniaturization, channel increases, price reductions, and high processing speeds. The application areas for laser scanners, such as industrial manufacturing, healthcare, civil engineering, entertainment, media, aerospace, and defense [18, 19], are expanding broadly and growing rapidly. In the surveying industry, application fields are expanding from aerial or ground surveys to autonomous surveys based on unmanned aerial vehicles (UAVs) and motor vehicles [20]. In particular, the laser scanner is expected to grow rapidly as an essential sensor for autonomous vehicles [21].

LiDAR is expected to be useful for road surface condition analysis because it can measure both backscattering intensity and the range between the sensor and the target. The intensity is regarded as auxiliary data in the surveying field. LiDAR sensors are mainly used for 3D surveying and object detection. Initially, LiDAR was developed to focus on improving point density by increasing the laser pulse frequency [22, 23]. There has been relatively little interest in the use of backscattering intensity data, although more information can be acquired about reflectivity of the target. Some studies have reported a strong correlation between backscattering intensity of a laser pulse and the spectral reflectance at the wavelength of the laser used $[24,25]$. Recently, with increasing interest in the use of backscattering intensity, multispectral LiDAR has been developed to simultaneously acquire both three-dimensional coordinates and types of land-cover information [26]. The detailed theories about LiDAR and backscattering intensity are covered in Background.

The aim of this study is investigation into the signal characteristics of laser backscattering intensity in order to detect frozen and wet surfaces on roads. For an experiment, an ice target, a wet surface, a dry surface, and roadside vegetation were scanned using a laser scanner. Then, signal characteristics were investigated to detect frozen and wet surfaces on the road. Finally, we explore the possibilities for detection or classification of road surface conditions.

\section{Background}

2.1. LiDAR. LiDAR is a system consisting of a laser scanner and a global positioning system/inertial navigation system (GPS/INS) [22, 27]. The laser scanner transmits laser pulses and receives backscattered laser pulses from the target. It is a simple principle to calculate the range between sensor and target using the difference between transmitted time and received time and the speed of light, as expressed in (1) and seen in Figure 1:

$$
\mathrm{R}=\frac{1}{2} \times c \times t_{L}
$$

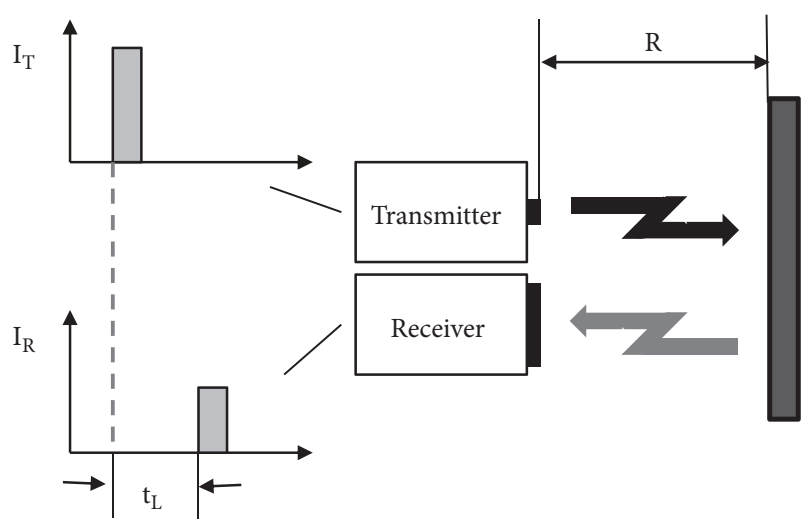

FIGURE 1: Time-of-flight ranging. $\mathrm{I}_{\mathrm{T}}$ and $\mathrm{I}_{\mathrm{R}}$ are the transmitted and received intensity, respectively. Adapted from Wehr and Lohr [27].

where $\mathrm{R}$ is the range between the sensor and the target surface, $t_{L}$ is the round-trip time of the laser pulse, and $c$ is the speed of light.

The laser scanner scans a three-dimensional area by changing the scanning direction rapidly and repeating the transmission and reception of laser pulses. The scanner simultaneously measures direction, round-trip time, and backscattering intensity of the laser pulses. The range resolution $(\Delta \mathrm{R})$ is determined according to the transmit-andreceive repetition period $\left(\Delta \mathrm{t}_{\mathrm{L}}\right)$ of the laser pulse, as shown in the following equation:

$$
\Delta \mathrm{R}=\frac{1}{2} \times c \times \Delta t_{L}
$$

When the scanner is moved by the platform, the position and attitude of the scanner are measured simultaneously using the GPS and INS. The direction and round-trip time are used to determine three-dimensional coordinates $(\mathrm{X}, \mathrm{Y}, \mathrm{Z})$ of target surfaces, combined with the position and attitude of the sensor from the GPS/INS.

2.2. Backscattering Intensity. Laser pulses are generated with a narrow wavelength width in the green, red, and nearinfrared regions. The wavelength of laser pulses affects the maximum range and the signal-to-noise ratio (SNR) [27]. The maximum range of the pulses is dependent on the wavelength of the laser used and the spectral reflectance of the target. The SNR depends on the wavelength owing to intensive background illumination, such as sunlight. SNR affects the accuracy of the range measurement. The laser scanner measures the intensity of the received laser pulse in addition to the round-trip time to estimate the effective maximum range of the laser pulse. The intensity is recorded as a relative digital number (DN) within the allowed radiometric resolution of the system [28]. Figure 2 shows the range correction factor of a laser scanner manufactured by Reigle as an example. Point A in Figure 2 represents a target with 20\% reflectivity which has a maximum range of only $50 \%$ of the one given in the laser specifications. 


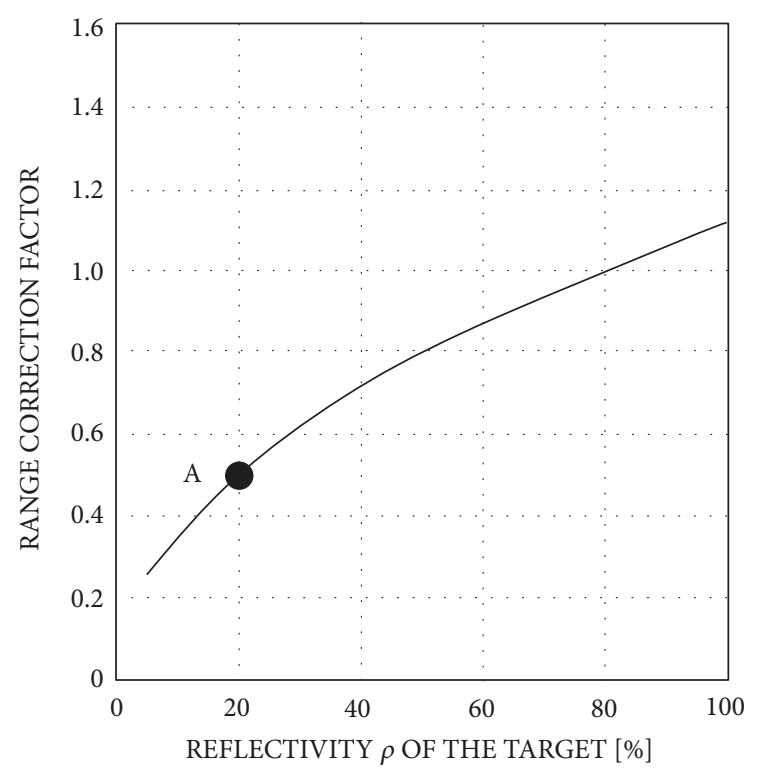

FIGURE 2: Range correction factor of a laser pulse based on reflectivity of the target. Adapted from Wehr and Lohr [27].

The intensity of the laser pulse at the receiver can be expressed with the following equation $[29,30]$ :

$$
I_{R}=I_{T} \times \frac{\cos \theta}{R^{2}} \times \rho \times \eta_{\text {atm }} \times C
$$

where $I_{R}$ is the received intensity, $I_{T}$ is the transmitted intensity, $R$ is the range from scanner to target, $\theta$ is the effective incidence angle (the angle between the direction of the laser pulse and the surface normal at the target), $\rho$ is the reflectance of the target, $\eta_{a t m}$ is an atmospheric attenuation constant, and $C$ is a sensor system constant factor. In (3), the reflectance of the target might be the only effective factor of the intensity when the measuring environments (such as geometric condition, weather, and instruments) are the same. It is necessary to know the spectral reflectance of the target to understand the characteristics of the backscattering intensity.

Various materials on the Earth's surface have their own spectral reflectance from chemical compositions and physical structures, which is called the spectral signature [31]. Figure 3 shows spectral reflectance curves (spectral signatures) of representative surface types for roads and roadsides, which were collected from the spectral library of Johns Hopkins University (JHU) and the United States Geological Survey (USGS) $[32,33]$. Ice has high reflectance (over $80 \%$ ), from 0.4 to $1.4 \mu \mathrm{m}$. Asphalt has continuously increasing reflectance, from $5 \%$ to $12 \%$ of the whole wavelength range. Water has about a 5\% reflectance in blue and green regions $(0.4-0.5$ $\mu \mathrm{m})$, and then reflectance decreases to about $0 \%$. Vegetation shows a small peak in the green $(0.55 \mu \mathrm{m})$ region and a rapid increase at the red edge (near $0.7 \mu \mathrm{m}$ ) and then high reflectance in the NIR region $(0.8-1.4 \mu \mathrm{m})$.

Spectral reflectance is also determined based on surface roughness compared to wavelength [31]. A smooth surface shows specular reflection, and a rough surface shows diffused reflection (Figure 4). In specular reflection, incident energy

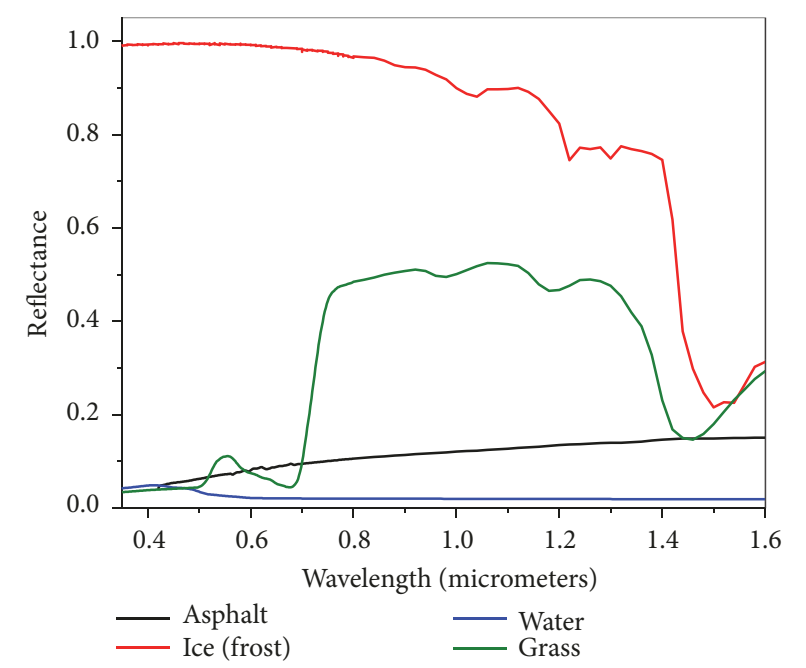

FIGURE 3: Spectral reflectance curves of asphalt, ice (frost), water, and grass (vegetation) collected from spectral libraries of JHU and USGS.

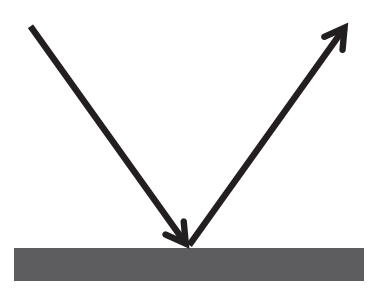

Specular reflection

(a)

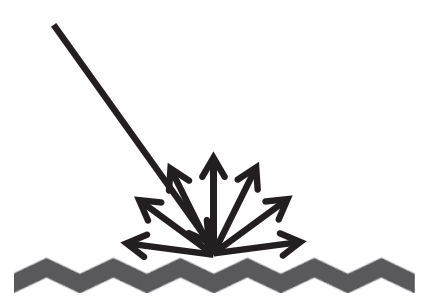

Diffused reflection

(b)
FIGURE 4: (a) Specular reflection on a smooth surface and (b) diffused reflection on a rough surface.

is reflected toward the incident direction with the same intensity. In diffused reflection, incident energy is reflected in dispersed directions, and the intensity of the energy returning to the sensor is weakened. Therefore, surface roughness affects the strength of the energy returning to the sensor.

One study reported the directional distribution of reflected energy by various conditions of asphalt surface (dry, water-covered, ice-covered, and snow-covered) using halogen illumination [34]. The strongest reflection backward, in the sensor direction, was when an asphalt surface is dry. The most of incident energy was reflected in the incident direction when the surface condition was covered with water or ice. Snow showed diffused reflectance, and energy was reflected by a rough surface in all directions with a similar intensity.

By this theory, laser backscattering intensity can be used to analyze road surface conditions. However, there have been limited such experiments. Therefore, it is necessary to investigate the possibility of detecting and classifying road surface conditions by analyzing the characteristics of backscattering intensity from a laser. 


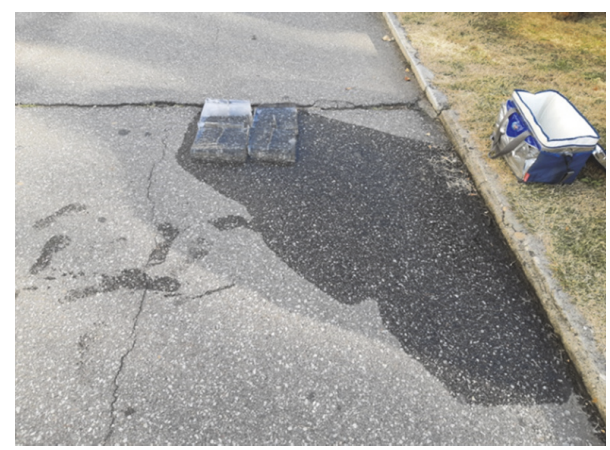

(a)

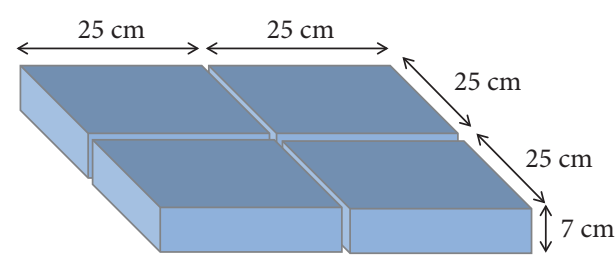

(b)

FIgURE 5: (a) Adjoining ice cubes as a target and asphalt road surface wet from melting ice. (b) The structure and size of the ice target.

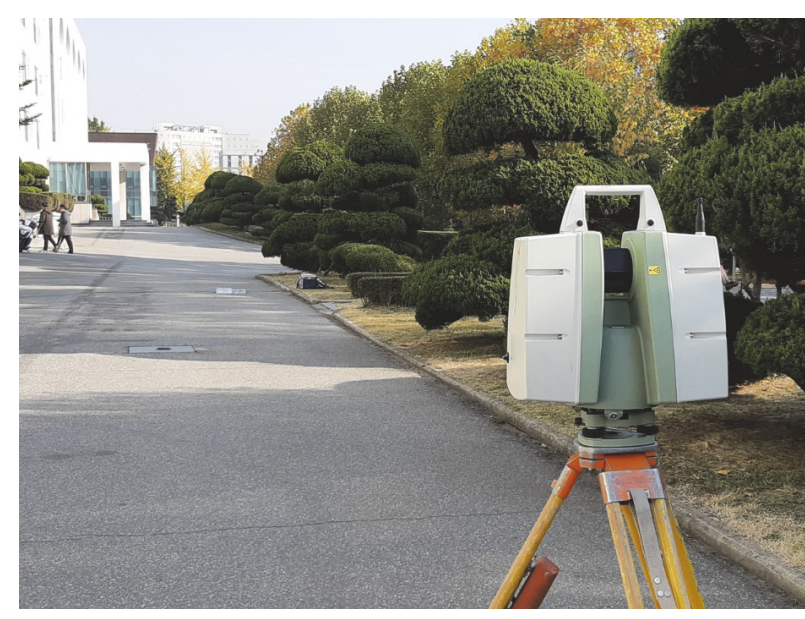

(a)

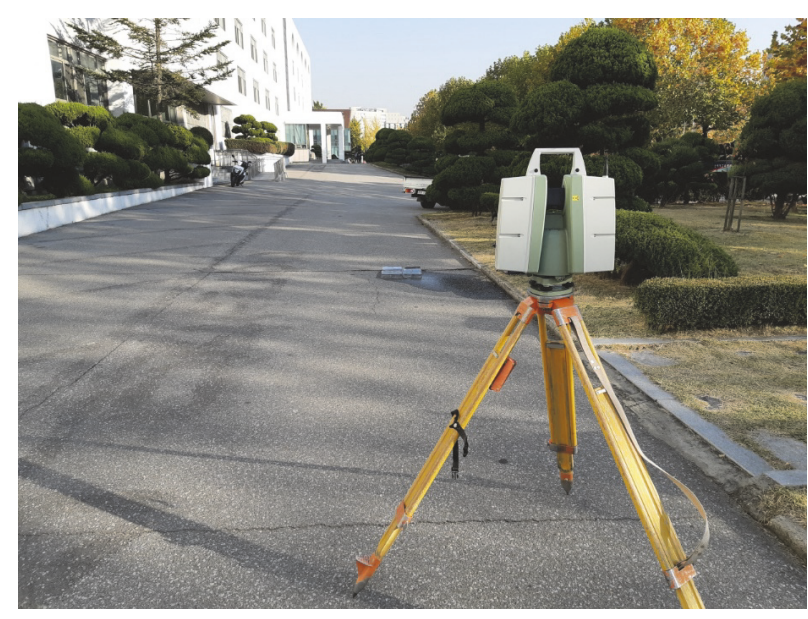

(b)

FIGURE 6: Laser scanning of the road surface and ice target at (a) $30 \mathrm{~m}$ and (b) $5 \mathrm{~m}$ distances.

TABLE 1: Specifications of the ScanStation C10 laser scanner.

\begin{tabular}{lc}
\hline Wavelength & $532 \mathrm{~nm}$ (green) \\
Laser class & $3 \mathrm{R}$ (IEC 60825-1) \\
Range & $300 \mathrm{~m} @ 90 \% ; 134 \mathrm{~m} @ 18 \%$ albedo \\
Spot (footprint) size & $4.5 \mathrm{~mm}$ within a $50 \mathrm{~m}$ distance \\
Point spacing & $1 \mathrm{~mm}$ minimum \\
Scan rate & Up to $50,000 \mathrm{pts} / \mathrm{sec}$ \\
Field of view & Horizontal: $360^{\circ}$, Vertical: $270^{\circ}$ \\
\hline
\end{tabular}

\section{Materials and Methods}

3.1. Laser Scanner. To measure laser backscattering intensity, this study used a ScanStation C10 laser scanner manufactured by Leica Geosystems, Switzerland. Table 1 shows the specifications of the laser scanner used. It has a 3R class green laser $(532 \mathrm{~nm})$ with a range of up to $300 \mathrm{~m}$. The scan rate is up to 50,000 points per second, the spot size is $4.5 \mathrm{~mm}$ at $0-50 \mathrm{~m}$, and the point spacing is at least $1 \mathrm{~mm}$.

3.2. Ice Target. An ice target was prepared by placing four ice cubes side by side on an asphalt road (Figure 5(a)). Each ice cube had a width of $25 \mathrm{~cm}$, a depth of $25 \mathrm{~cm}$, and thickness of $7 \mathrm{~cm}$ (Figure 5(b)). The total size of the target was $50 \mathrm{~cm} \times 50 \mathrm{~cm} \times 7 \mathrm{~cm}$. The four ice cubes touched, but there was actually a gap between them. There was an opaque part at the upper left of the ice target, as shown in Figure 5(a). Black ice on a road surface has a thickness of only several millimeters. However, considering the temperature and production conditions, the ice cubes were made $7 \mathrm{~cm}$ thick. After the ice cubes were placed, a wet asphalt surface formed from melting ice.

3.3. Laser Backscattering Intensity Measurements. Horizontal distances between the laser scanner and the ice target were $30 \mathrm{~m}$ and $5 \mathrm{~m}$, as seen in Figure 6. Scanning angle was set to a range of $45^{\circ}$ horizontally, centered on the target, and $-45^{\circ}$ to $+45^{\circ}$ vertically. The experiment site was an asphalt road at Inha University, Incheon, Republic of Korea. Laser scanning data were acquired between 15:09 and 16:05 hours, local time, on 2 November 2018. The solar elevation angle decreased from $25^{\circ}$ to $16^{\circ}$ during measurement, and the site was shadowed by surrounding trees. Temperature was about $15^{\circ} \mathrm{C}$, and humidity was $63 \%$. Figure 7 shows the geometric configuration for laser scanning. The height of the sensor was 


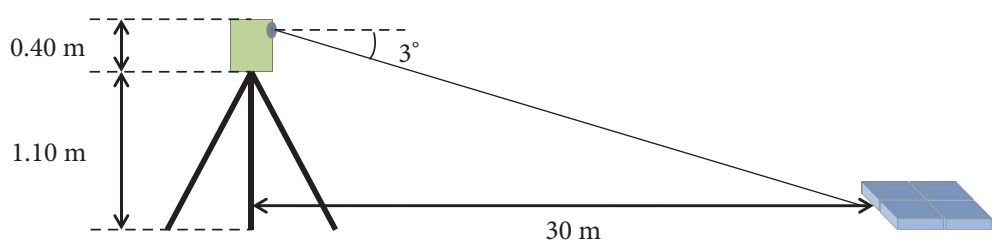

(a)

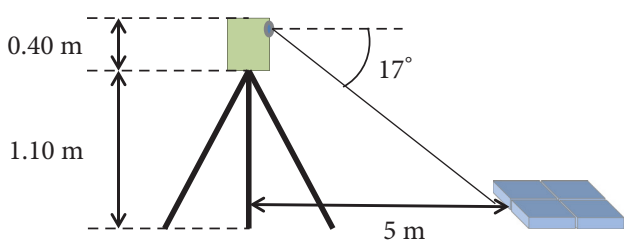

(b)

FIGURE 7: Geometric configuration for laser scanning at (a) $30 \mathrm{~m}$ and (b) $5 \mathrm{~m}$ distances.

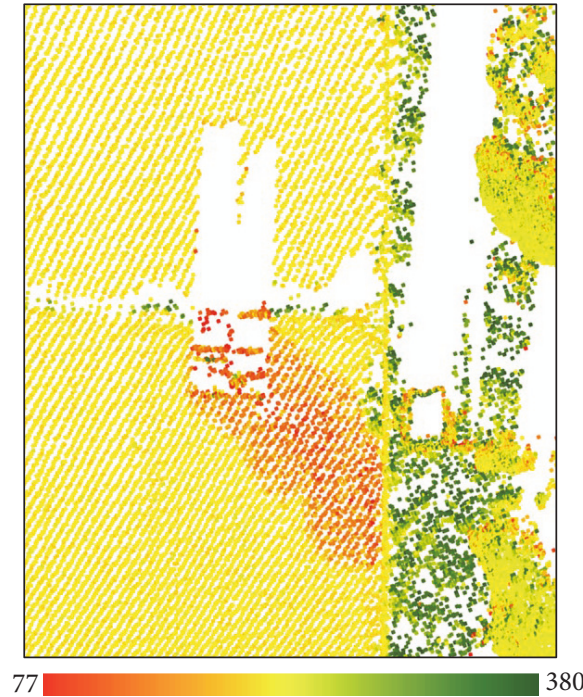

(a)

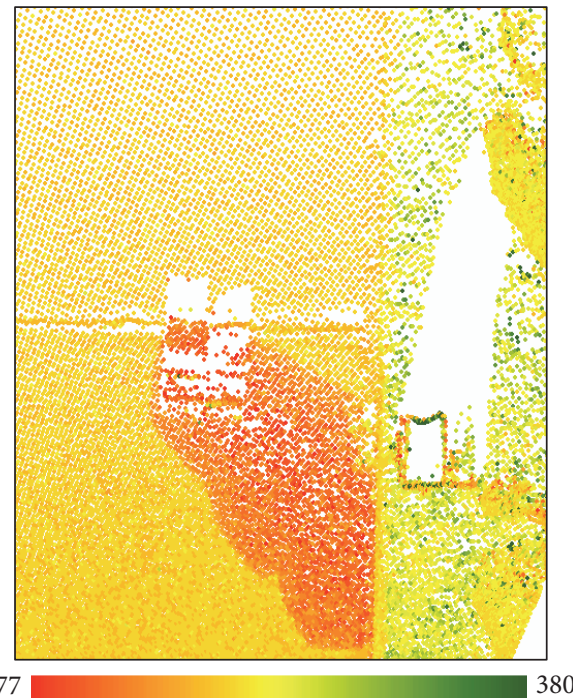

(b)

FIGURE 8: Nadir view of backscattering intensity at (a) $30 \mathrm{~m}$ and (b) $5 \mathrm{~m}$ sensor-target distances.

about $1.5 \mathrm{~m}$. Around the ice target, the vertical incidence angle of the laser pulse was about $3^{\circ}$ and $17^{\circ}$ for the $30 \mathrm{~m}$ and $5 \mathrm{~m}$ distances, respectively.

\section{Results}

4.1. Laser Scanning Data. Laser scanning data were obtained in the form of a point cloud, as shown in Figure 8. The point cloud data were converted to ASCII XYZI format. In the XYZI format, each point is separated by a row, and each row consists of four columns, which are three-dimensional coordinates $(\mathrm{X}, \mathrm{Y}, \mathrm{Z})$ plus backscattering intensity, where intensity is a relative $D N$ value with a 12-bit depth [28]. Figures $8(a)$ and 8(b) show point clouds of the laser backscattering intensity data obtained at $30 \mathrm{~m}$ and $5 \mathrm{~m}$ distances, respectively, on a two-dimensional plane ( $\mathrm{X}$ and $\mathrm{Y}$ ), viewed from the nadir. For interpretation by eye, the backscattering intensity of each point is colored red to green between a minimum value of 77 and a maximum value of 380 . At the target-sensor distance of $30 \mathrm{~m}$, point spacing was $1.25 \mathrm{~cm}$ in the scanning (vertical) direction and $5 \mathrm{~cm}$ in the rotation (horizontal) direction. When the target-sensor distance was $5 \mathrm{~m}$, point spacing was $1.25 \mathrm{~cm}$ in the scanning (vertical) direction and 2.08 $\mathrm{cm}$ in the rotation (horizontal) direction. It is possible to analyze the characteristics of laser backscattering data from two aspects: missing backscattering data and backscattering intensity based on road surface conditions.

4.2. Missing Laser Backscattering Signals. There are some blank areas without laser backscattering signal in Figure 8. These are the top surface of the ice target, some parts of the vegetation area, and the areas occluded by the ice target and a box.

The top surface of the ice target could not be scanned. The primary reason might be specular reflection from the smooth surface. Spectral reflectance and effective incidence angle are major factors in backscattering intensity under the same geometric conditions (see (3)). The effective incidence angle is affected by the target's structure. Ice has a very high reflectance in the visible and NIR region, in theory, but no backscattering signal was observed in the experiment. The reasons for the missing signals might be absorption by moisture and specular reflection by a smooth surface, as shown as Figure 4. Absorption of moisture was not considered a reason, because the sidewall and the opaque parts of the ice showed backscattering signals under the same moisture conditions. Except for absorption, specular reflection can be considered a key factor in backscattering. Therefore, the missing backscattering signals from the top of the ice were caused by specular reflection from smoothness. 
TABLE 2: Statistics of laser backscattering intensity for surface types at the $30 \mathrm{~m}$ distance.

\begin{tabular}{lcccc}
\hline Surface Type & Vegetation & Dry Asphalt & Wet Asphalt & Ice (Sidewall) \\
\hline No. of sample points & 208 & 296 & 195 & 143.19 \\
Mean & 328.86 & 208.09 & 16.94 & 157.51 \\
Standard deviation & 95.51 & 7.09 & 93 & 70.15 \\
Minimum & 196 & 190 & 176 & 77 \\
Maximum & 634 & 236 & & 852 \\
\hline
\end{tabular}

TABLE 3: Statistics of laser backscattering intensity for surface types at the $5 \mathrm{~m}$ distance.

\begin{tabular}{lcccc}
\hline Surface Type & Vegetation & Dry Asphalt & Wet Asphalt & Ice (Sidewall) \\
\hline No. of sample points & 471 & 535 & 323 & 131.49 \\
Mean & 244.43 & 206.56 & 20.24 & 148 \\
Standard deviation & 21.67 & 7.96 & 82 & 66.74 \\
Minimum & 200 & 189 & 178 & 78 \\
Maximum & 372 & 233 & & 835 \\
\hline
\end{tabular}

In real applications, it is possible to detect black ice by using a phenomenon where signals are suddenly missing during periodic scanning of the road surface.

There are two big blank areas; those are the asphalt surface behind the ice target and the grass behind the box, respectively. They are areas occluded by the ice target and the box. The blank areas are longer at the $30 \mathrm{~m}$ distance than at the $5 \mathrm{~m}$ distance due to incidence angles. The laser pulses have a smaller incidence angle at the $30 \mathrm{~m}$ distance, as shown in Figure 7.

Some small blank areas of vegetation might be caused by the incidence angle. The ratio of blank areas is higher at 30 $\mathrm{m}$ than at $5 \mathrm{~m}$. The laser pulses might be blocked by some leaves of grass with small effective incidence angles, because they have a vertical structure.

\subsection{Backscattering Intensity by Road Surface Conditions.} Laser pulses were backscattered from the surface of the scanning area except for missed or occluded areas. Some laser pulses were backscattered at the sidewalls facing the sensor and at the gaps between the ice cubes, although most of the missing backscattering signals were from the top of the ice target. The backscattering signal was concentrated at the upper left of the ice target in Figure 8. It is the opaque part of the ice target, as shown in Figure 5(a). There are more points from the ice target occurring at the $5 \mathrm{~m}$ distance, compared to $30 \mathrm{~m}$, because more opaque parts might be seen from a bigger incidence angle.

In Figure 8, colored backscattering intensity looks like it is classified by road surface conditions, including vegetation beside the road. Some parts of the ice target and the wet asphalt are shown in red with a low value. Dry asphalt is shown as orange, and yellow indicates mid-range intensity. Vegetation at the roadside is shown in green at high intensity. These are caused by spectral reflectance of the target in the wavelength of the laser used, where spectral reflectance is affected by absorption, transmittance, and surface roughness [31]. The sum of absorbed, transmitted, and reflected energy is equivalent to the incident energy. Reflectance is related to surface roughness, as mentioned above.

In Tables 2 and 3, statistics of backscattering intensity for each surface type are shown based on sensor-target distance. Sample points were collected from each surface type, and then statistics for backscattering intensity were calculated (mean, standard deviation, minimum, and maximum). The mean values for surface types have a similar pattern, with spectral reflectance in the green region $(0.5-0.6 \mu \mathrm{m})$ of Figure 3 and from a previous study [34]. Mean values were in the following order: vegetation, dry asphalt, wet asphalt, and sidewall of the ice. High backscattering intensities for vegetation could be explained by the wavelength of the laser and the surface roughness. The laser scanner used a 532 $\mathrm{nm}$ equivalent green region where spectral reflectance of vegetation is higher than other surface types. Vegetation has the highest surface roughness among the surface types, which is a reason for higher backscattering intensity than from other surface types. Dry asphalt showed higher backscattering intensity than wet asphalt due to the absorption of electromagnetic energy by moisture. Dry asphalt also showed a higher backscattering intensity than the sidewall of the ice, because it has relatively higher roughness, even though asphalt has a lower spectral reflectance than ice. Additionally, the backscattering intensity of the sidewall facing the sensor was low due to transmission from clear ice (a crystal), although the spectral reflectance of ice is known to be very high in the visible-NIR region.

The mean intensities are lower at $5 \mathrm{~m}$ than at $30 \mathrm{~m}$. The reason might be that received intensity decreases with an increasing effective incidence angle by the cosine function in (3). Vegetation shows a relatively bigger decrease than other surface types. The sample points were collected from grass, where leaves are distributed vertically. The vertical leaves might be able to strongly reflect and block the laser pulse incident horizontally. This might be the same reason for small blanks in the vegetation. The dispersed laser pulse might be weakened when penetrating the bottom through 


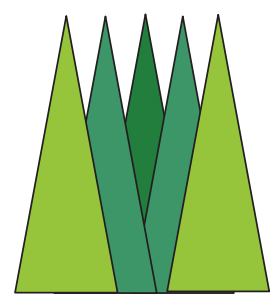

(a)

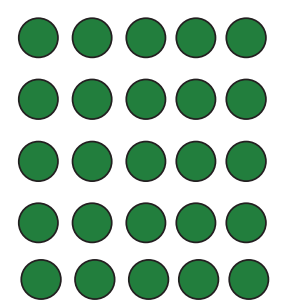

(b)

FIGURE 9: Difference of homogeneity for vertical grass leaves when a sensor points (a) in a horizontal direction and (b) at the nadir.

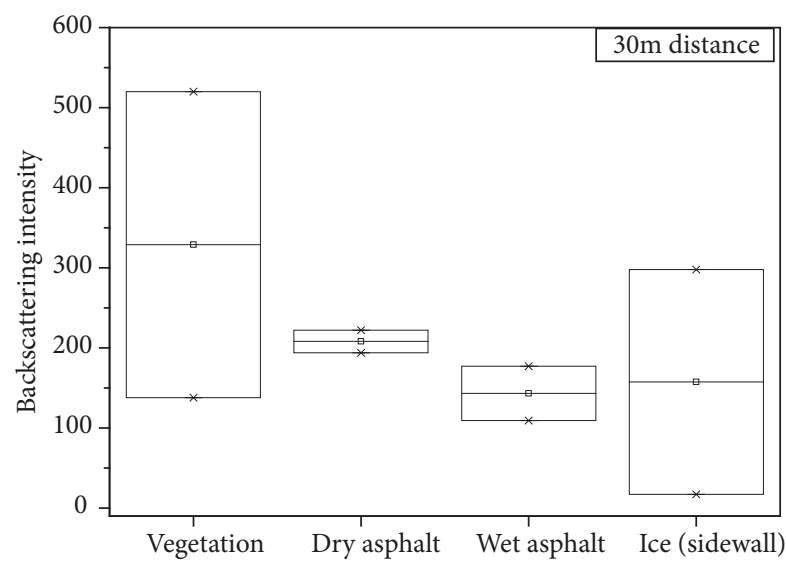

(a)

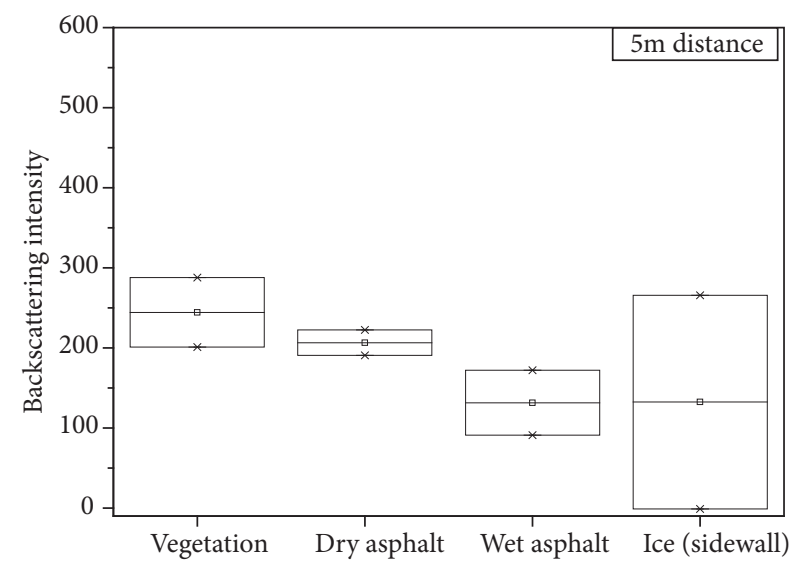

(b)

FIGURE 10: Ranges of $95 \%$ distribution for laser backscattering intensity in each surface type: (a) $30 \mathrm{~m}$ distance and (b) $5 \mathrm{~m}$ distance. Backscattering intensity of ice is the signal from the sidewall face to the sensor direction.

the leaves when the incidence angle is big. Therefore, sensortarget geometry and physical structure of the target might be reasons for the decreased mean in backscattering intensity at $5 \mathrm{~m}$, compared to $30 \mathrm{~m}$.

Standard deviation of vegetation decreased at the $5 \mathrm{~m}$ distance, although the other surface types showed similar values. The reason might be increasing homogeneity by an increasing incidence angle, such as in Figure 9. Vertical leaves of grass might show higher homogeneity from a sensor that looks at the nadir, rather than a horizontal direction.

It is necessary to check whether the surface condition can be classified by statistical analysis. Mean and standard deviation are generally considered as distribution ranges for classification. Classes were confused when the distribution ranges were overlapped by a high standard deviation, even though the means of the two classes were different. Figure 10 shows the ranges of mean $\pm 2 \times$ standard deviation using the statistics for each surface type. Wet asphalt and dry asphalt are classified clearly by the $95 \%$ range. However, the ranges for vegetation and ice (sidewall) are broad and overlap other surface types. The wide intensity range of vegetation was caused by multiple backscattering from penetrating laser pulses. For the ice, some energy of a laser pulse might be backscattered from the ice surface, and the remaining energy might be backscattered from the asphalt through the ice target. For the vegetation, some energy of a laser pulse might reach through the leaves. These were common phenomena in the observations at both $30 \mathrm{~m}$ and $5 \mathrm{~m}$ distances.

\section{Discussion}

The results showed that backscattered points were missing for the top surface of the ice target. The mean backscattering intensity of dry asphalt, wet asphalt, and vegetation, except for the ice target, showed a pattern similar to the spectral reflectance at the wavelength of the laser used. The difference in backscattering intensity according to the sensor-target distance is considered to depend on the incidence angle and the structure of the target. The differences are small for a twodimensional road surface but relatively large for the complex three-dimensional vegetation.

The condition of the road surface could be classified as a dry surface, a wet surface, and a thin ice sheet (such as black ice) by using laser backscattering intensity within the road. There were limitations to classifying the condition of the road surface, including vegetation and the sidewall of thick ice cubes, using intensity. However, an ice sheet is usually several millimeters thick on the road surface, and vegetation has height and a rough surface. The road area also might be extracted with texture or auxiliary data. Consequently, laser backscattering intensity could be used to detect frozen and wet road surface conditions.

For applications, the platforms for operating a laser scanner can be either fixed or mobile. A mobile platform can represent a vehicle. In the near future, it should be possible to acquire data easily through LiDAR, which is a key sensor in autonomous vehicles. There is a limit to this, in that the road 
surface condition will be scanned from a driven vehicle. A fixed platform can be like the facilities above, or on the side of the road, such as traffic lights or an intelligent transportation system (ITS). The fixed platform has advantages in that it is possible to monitor the road surface condition, in addition to falling obstacles, through continuous observation of a predetermined area. However, many stations are needed to acquire spatially continuous information, which may be inefficient in terms of cost. Therefore, it is necessary to maximize efficiency by optimizing the installation site using information about frequent occurrences of frozen road surfaces and hydroplaning.

\section{Conclusions}

In this study, we investigated the possibility of extracting driving safety information, such as identifying frozen, dry, and wet conditions on a road surface, from laser backscattering intensity data. Laser backscattering intensity was measured for an ice target, dry asphalt, wet asphalt, and vegetation from ranges of $5 \mathrm{~m}$ and $30 \mathrm{~m}$. As a result, the backscattering signal was missing from the top of the ice due to specular reflection. A wet asphalt surface showed lower intensity than a dry asphalt surface. Therefore, it is possible to detect frozen and wet road surfaces versus dry conditions. The laser scanner can contribute in the future to improving safety for drivers by enabling night observation. Further studies are needed using a smaller and cheaper laser scanner. It is also necessary to check the possibility of applying the method in various environments.

\section{Data Availability}

The laser scanning data used to support the findings of this study are available from the corresponding author upon request.

\section{Conflicts of Interest}

The authors declare no conflicts of interest regarding the publication of this paper.

\section{Acknowledgments}

This research was supported by a grant [18SIUE-B148326-01] from Satellite Information Utilization Center Establishment Program by Ministry of Land, Infrastructure and Transport of Korean government, and by a grant [2019-0145] from the Major Project by Korea Institute of Civil Engineering and Building Technology. The authors acknowledge Neighbor Systems Inc., Seoul, Republic of Korea, for supporting this study with the laser scanner instrument used.

\section{References}

[1] H. Yasuura, C. Kyung, Y. Liu, and Y. Lin, Smart Sensors at the IoT Frontier, Springer International Publishing, Cham, 2017.

[2] P. A. Pisano, L. C. Goodwin, M. A. Rossetti, and U. S, "highway crashes," in Proceedings of 24th Conference on IIPS, no. 8.1, 2008.
[3] M.-P. Andreescu and D. B. Frost, "Weather and traffic accidents in Montreal, Canada," Climate Research, vol. 9, no. 3, pp. 225230, 1998.

[4] S. Kim, Y. Jang, S. Kim, D. Min, H. Na, and J. Choi, "A Study on the Effects of Factors of Traffic Accidents Caused by Frozen Urban Road Surfaces in the Winter," Journal of the Korean Society of Road Engineers, vol. 17, no. 2, pp. 79-87, 2015.

[5] E. W. Black, "Wikipedia and academic peer review," Online Information Review, vol. 32, no. 1, pp. 73-88, 2008.

[6] K. Fujimura and T. Skamoto, "Road Surface Sensor," Fujitsu TEN Tech. Journal, vol. 1, no. 1, pp. 64-72, 1988.

[7] F. Rios-Gutierrez and M. A. Hasan, "Survey and evaluation of ice/snow detection technologies," Final Report of Northland Advanced Transportation Systems Research Laboratories, University of Minnesota Duluth, vol. 18, pp. 1-23, 2003, http://www .d.umn.edu/natsrl/documents/FY2003reports/IRID_2003.pdf (accessed on 18 Nov. 2018).

[8] G. Pan, L. Fu, R. Yu, and M. Muresan, "Winter road surface condition recognition using a pretrained deep convolutional network," in Proceedings of Transportation Research Board 97th Annual Meeting, 2018, https://arxiv.org/abs/1812.06858v1.

[9] P. Jonsson, J. Casselgren, and B. Thörnberg, "Road surface status classification using spectral analysis of NIR camera images," IEEE Sensors Journal, vol. 15, no. 3, pp. 1641-1656, 2015.

[10] Vaisala, DSC111 Remote Surface State Sensor, 2019, http://www .vaisala.com/en/products/surfacesensors/Pages/DSC111.aspx (accessed on 16 Mar. 2019).

[11] L. Colace, F. Santoni, and G. Assanto, "A near-infrared optoelectronic approach to detection of road conditions," Optics and Lasers in Engineering, vol. 51, no. 5, pp. 633-636, 2013.

[12] L. Ewan, A. Al-Kaisy, and D. Veneziano, "Remote Sensing of Weather and Road Surface Conditions," Transportation Research Record, vol. 2329, no. 1, pp. 8-16, 2013.

[13] M. Kutila, M. Jokela, J. Burgoa, A. Barsi, T. Lovas, and S. Zangherati, "Optical roadstate monitoring for infrastructureside co-operative traffic safety systems," in Proceedings of the IEEE Intelligent Vehicles Symposium, pp. 620-625, IEEE, Eindhoven, The Netherlands, June 2008.

[14] D. F. Huber, L. Denes, M. Hebert, M. Gottlieb, B. Kaminsky, and P. Metes, "A spectro-polarimetric imager for intelligent transportation systems," in Proceedings of the Intelligent Transportation Systems, pp. 94-102, USA, October 1997.

[15] Lufft, MARWIS-UMB - Mobile Advanced Road Weather Information Sensor, 2018, https://www.lufft.com/products/road-runway-sensors-292/marwis-umb-mobile-advanced-road-weather-information-sensor-2308/ (accessed on 20 Nov. 2018).

[16] A. Bystrov, E. Hoare, T. Tran, N. Clarke, M. Gashinova, and M. Cherniakov, "Automotive system for remote surface classification," Sensors, vol. 17, no. 745, pp. 1-18, 2017.

[17] I. Gresham, N. Jain, T. Budka et al., "A compact manufacturable 76-77-GHz radar module for commercial ACC applications," IEEE Transactions on Microwave Theory and Techniques, vol. 49, no. 1, pp. 44-58, 2001.

[18] Allied Market Research, "3D scanning market by type, range, service, and application," Global Opportunity Analysis and Industry Forecast, 2018, https://www.alliedmarketresearch .com/3D-scanning-market (accessed on 16 Mar. 2019).

[19] Global Market Insight, "3D scanning market size by range, by product, by application, industry analysis report, regional outlook, growth potential, price trends," Competitive Market Share and Forecast, 2017, https://www.gminsights.com/industryanalysis/3d-scanning-market (accessed on 16 Mar 2019). 
[20] Y. Lin, J. Hyyppä, and A. Jaakkola, "Mini-UAV-borne LIDAR for fine-scale mapping," IEEE Geoscience and Remote Sensing Letters, vol. 8, no. 3, pp. 426-430, 2011.

[21] J. Shi, J. Guo, M. Kagami, P. Suni, and O. Ziemann, "Photonic technologies for autonomous cars: feature introduction," Optics Express, vol. 27, no. 5, pp. 7627-7628, 2019.

[22] E. P. Baltsavias, "Airborne laser scanning: Basic relations and formulas," ISPRS Journal of Photogrammetry and Remote Sensing, vol. 54, no. 2-3, pp. 199-214, 1999.

[23] S. Kaasalainen, T. Lindroos, and J. Hyyppä, “Toward hyperspectral lidar: measurement of spectral backscatter intensity with a supercontinuum laser source," IEEE Geoscience and Remote Sensing Letters, vol. 4, no. 2, pp. 211-215, 2007.

[24] J.-S. Yoon, J.-I. Shin, and K.-S. Lee, "Land cover characteristics of airborne LiDAR intensity data: A case study," IEEE Geoscience and Remote Sensing Letters, vol. 5, no. 4, pp. 801-805, 2008.

[25] S. Kaasalainen, E. Ahokas, J. Hyyppä, and J. Suomalainen, "Study of surface brightness from backscattered laser intensity: Calibration of laser data," IEEE Geoscience and Remote Sensing Letters, vol. 2, no. 3, pp. 255-259, 2005.

[26] S. Morsy, A. Shaker, and A. El-Rabbany, "Multispectral LiDAR data for land cover classification of urban areas," Sensors, vol. 17, no. 958, pp. 1-21, 2017.

[27] A. Wehr and U. Lohr, "Airborne laser scanning-an introduction and overview," ISPRS Journal of Photogrammetry and Remote Sensing, vol. 54, no. 2-3, pp. 68-82, 1999.

[28] G. Jóźków, “Terrestrial laser scanning data compression using JPEG-2000," PFG - Journal of Photogrammetry, Remote Sensing and Geoinformation Science, vol. 85, no. 5, pp. 293-305, 2017.

[29] Q. Ding, W. Chen, B. King, Y. Liu, and G. Liu, "Combination of overlap-driven adjustment and Phong model for LiDAR intensity correction," ISPRS Journal of Photogrammetry and Remote Sensing, vol. 75, pp. 40-47, 2013.

[30] A. G. Kashani, M. J. Olsen, and A. J. Graettinger, "Laser scanning intensity analysis for automated building wind damage detection," in Proceedings of the 2015 ASCE International Workshop on Computing in Civil Engineering, IWCCE 2015, pp. 199-205, USA, 2015.

[31] J. R. Jensen, Remote Sensing of the Environment: An Earth Resource Perspective, Pearson, Upper Saddle River, NJ, USA, 2000.

[32] A. M. Baldridge, S. J. Hook, C. I. Grove, and G. Rivera, "The ASTER spectral library version 2.0," Remote Sensing of Environment, vol. 113, no. 4, pp. 711-715, 2009.

[33] Jet Propulsion Laboratory, "ECOSTRESS Spectral LibraryVersion 1.0," https://speclib.jpl.nasa.gov/ (accessed on 16 Mar 2019).

[34] J. Casselgren, M. Sjödahl, and J. LeBlanc, "Angular spectral response from covered asphalt," Applied Optics, vol. 46, no. 20, pp. 4277-4288, 2007. 


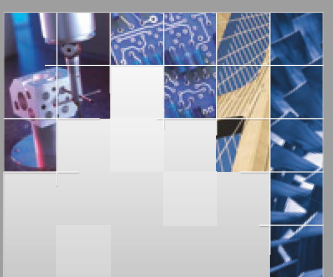

\section{Enfincering}
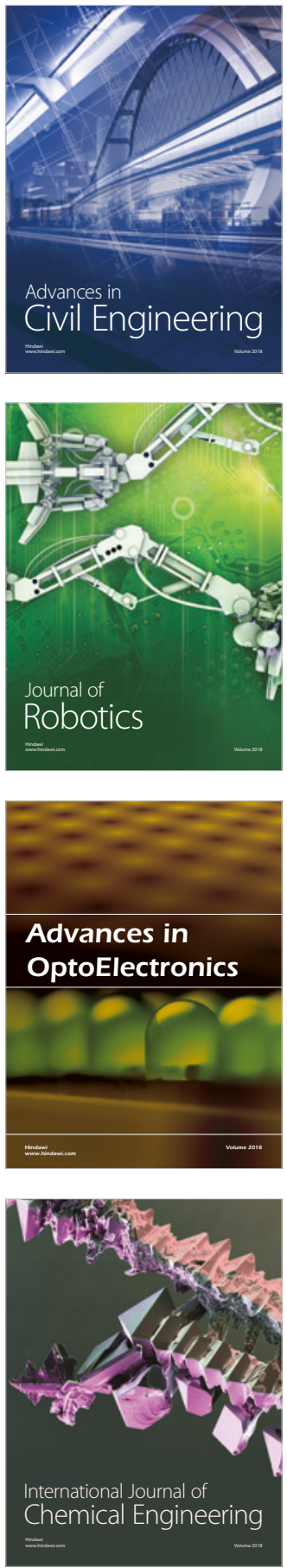

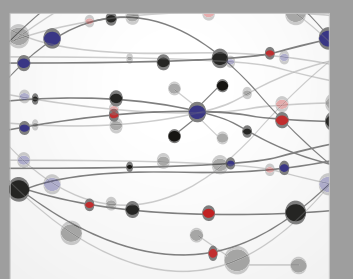

\section{Rotating \\ Machinery}

The Scientific World Journal

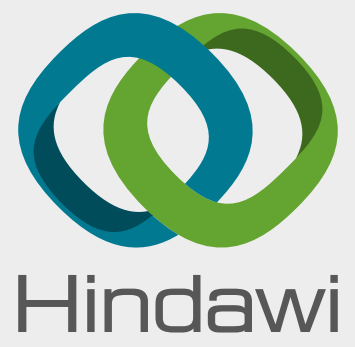

Submit your manuscripts at

www.hindawi.com
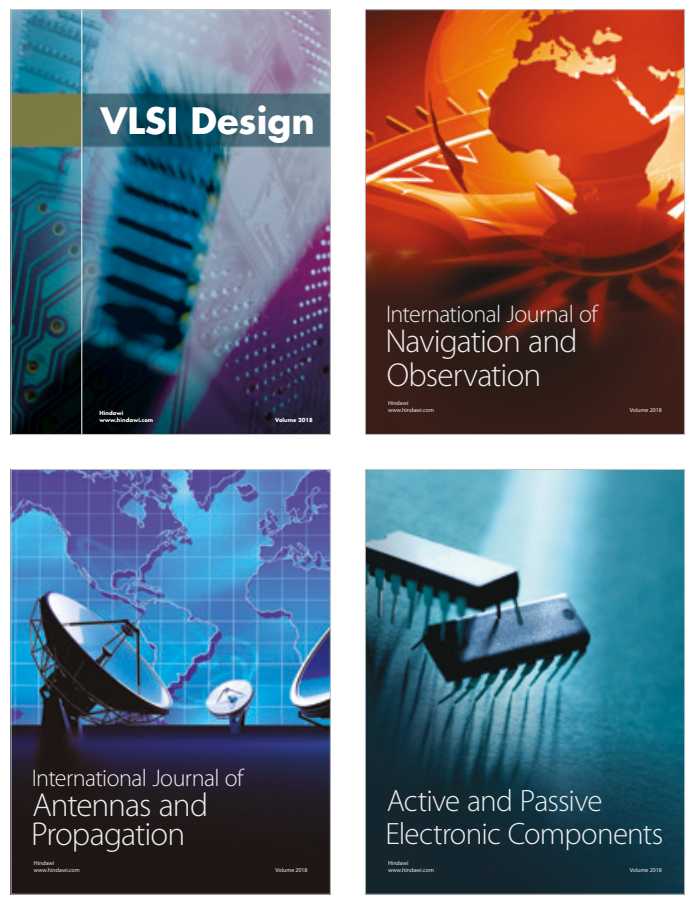
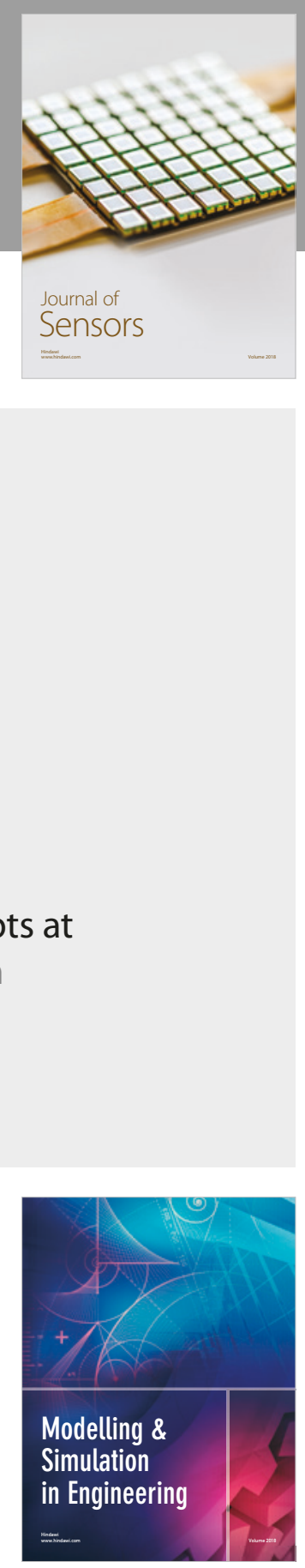

\section{Advances \\ Multimedia}
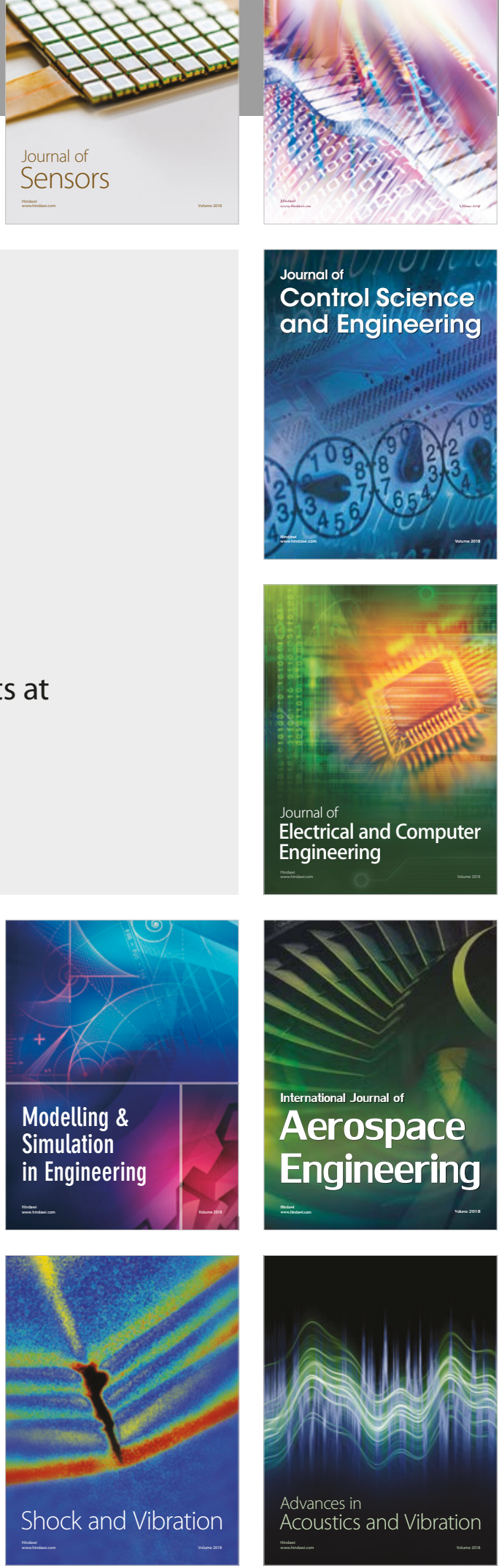